\title{
Ethnicity does not account for differences in the health-related quality of life of Turkish, Moroccan, and Moluccan elderly in the Netherlands
}

Ilona Verhagen*, Wynand JG Ros, Bas Steunenberg and Niek J de Wit

\begin{abstract}
Background: Data on how different groups of elderly immigrants perceive health-related quality of life (HRQOL) is scarce and research on the influence of ethnicity on HRQOL across ethnic groups is missing. Measuring HRQOL may help to detect cross-cultural differences and to decide whether ethnic-specific health and prevention programmes are required to improve HRQOL. We investigated differences in HRQOL among three elderly immigrant populations with a special focus on the contribution of ethnicity, in addition to other well-known determinants, to HRQOL.

Methods: Data were collected between October 2011 and July 2012 as part of the project entitled "Stem van de oudere migrant", a quasi-experimental study in the Netherlands focussing on health of immigrant elderly. A survey was conducted among 201 elderly (aged 55 years and older) Moroccans (98), Turks (69), and Moluccans (34). HRQOL was assessed using the SF-12, measuring physical and mental health composite scores (PCS resp. MCS). Chi-square tests and ANOVAs were performed for group comparison. Hierarchical multiple linear regressions were conducted to examine whether ethnicity uniquely contributed to the observed variance in $\mathrm{HRQOL}$ when multimorbidity, Ioneliness, socio-demographics, and acculturation were taken into account.

Results: Moroccans had the lowest scores on PCS (34.3 \pm 31.4$)$ and MCS (42.1 \pm 27.0$)$, followed by Turks (45.7 \pm 27.0 for PCS and $54.7 \pm 22.2$ for MCS), and Moluccans (71.7 \pm 21.2 for PCS and $74.4 \pm 22.1$ for MCS). Ethnicity was not independently associated with PCS and MCS scores, in contrast to loneliness (PCS $\beta-0.461, p<0.001$ and MCS $\beta$ $-0.435, p<0.001$ ) and multimorbidity (PCS $\beta-0.380, p<0.001$ and MCS $\beta-0.398, p<0.001$ ). Gender was independently associated with PCS $(\beta 0.148, p=0.026)$ and attachment to Dutch culture with MCS $(\beta 0.144$, $p=0.029$ ).
\end{abstract}

Conclusions: The lower level of HRQOL reported by elderly immigrant populations was affected by multimorbidity and loneliness but not ethnicity. Similar to native elders, interventions aiming at improving HRQOL for immigrant elderly should focus on loneliness and (mental and physical) disease. Finally, health literacy deserves attention to maintain health.

Trial registration: ISRCTN89447795

Keywords: Health-related quality of life, Elderly immigrants, Ethnicity, Multimorbidity, Loneliness

\footnotetext{
* Correspondence: i.verhagen-3@umcutrecht.nl

Julius Center for Health Sciences and Primary Care, University Medical Center Utrecht, Mailbox 85500, 3508 GA Utrecht, the Netherlands
} 


\section{Introduction}

The population in western countries is ageing and becoming more ethnically diverse. The health status [1-5] and health-related quality of life (HRQOL) [1,6] of immigrant elderly is often less favourable compared to native elderly. So far, data on how different groups of elderly immigrants perceive HRQOL are scarce, and research on the influence of ethnicity on HRQOL across ethnic groups is lacking. Measuring HRQOL may help to detect cross-cultural differences and to determine the necessity for ethnicity-specific health and prevention programmes to improve HRQOL.

Optimal health for the elderly is not a goal, as such, but it enables this population to maintain their independence, mobility and participation in life activities, as well as to respond to the challenges of old age [7]. The consequences of impaired health, rather than the absence of disease, mainly determine the quality of life for the elderly. Therefore, HRQOL is the most adequate parameter of well-being in older populations.

Loneliness, chronic health conditions, and multiple morbidities are well-known determinants of HRQOL [8-10]. Gender has been reported as a factor determining the HRQOL of native and immigrant elders [10-12]. Other factors that have been associated with HRQOL in immigrant elderly are educational level and co-morbidity [1]. Differences in HRQOL between foreign- and nativeborn elderly suggest that ethnicity may be another factor associated with HRQOL.

In the present study, we assessed differences in HRQOL among different ethnic groups of immigrant elders and whether, in addition to established determinants of HRQOL, such as multimorbidity, loneliness, socio-demographics, and acculturation, ethnicity contributed independently to HRQOL.

\section{Methods \\ Study population}

The study population consisted of a sample of elderly Moroccans, Turks, and Moluccans who participated in the project entitled "Stem van de oudere migrant" [13]. This quasi-experimental study aimed to assess the effectiveness of community health workers (CHWs) in improving access to health care facilities. The design and aims of that study are described elsewhere [13].

Turks and Moroccans immigrated to the Netherlands in the 1960s and early 1970s in pursuit of work and, later on, for family reunification or family formation. Moluccans, former soldiers in the Dutch colonial army, and their families were transferred to the Netherlands in 1951 after the decolonisation of Indonesia and were housed in resettlement camps in remote areas. Also included in the study were descendants of Moluccan immigrants who were born between 1951 and 1957 and raised in the Netherlands in the relatively isolated camps where the living situation, language and customs resembled those of the country of origin. Eligible participants were aged 55 years or older, living independently and currently not being treated for severe psychiatric disorders. Participants were recruited through CHWs. Details of the recruitment procedures have been described elsewhere [13].

\section{Data collection}

Data were collected between October 2011 and July 2012 by means of a structured personal interview conducted at home or at another location chosen by the participant. Trained interviewers from similar ethnic backgrounds verbally administered all questionnaire items by reading aloud the questions and the response options in the language of the participant. Standardised, translated versions of the questionnaire were used to minimise the variation in results and ensure the compatibility of interviews across all interviewers.

\section{Ethical principles}

All the participants provided oral or written informed consent. The Medical Ethics Committee of the University Medical Center Utrecht (UMCU) did not consider the study to be subject to the Medical Research Involving Human Subjects Act (WMO in Dutch); therefore, full ethical assessment was not required. The study is registered with the ISRCTN register: ISRCTN89447795.

\section{Outcome, determinants, and measurements}

The primary outcome was self-reported HRQOL, measured with the Short Form-12 (SF-12) [14]. The SF-12 is a widely used instrument to measure physical and mental aspects of HRQOL. The SF-12 is comprised of 12 items and yields 8 domain scores Physical Functioning; Role Physical, Bodily Pain, General Health, Vitality, Social Functioning, Role Emotional, and Mental Health that can be condensed into physical and mental health summary scores respectively the Physical Component Summary (PCS) and the Mental Component Summary (MCS).

We assessed the following determinants:

Ethnicity was determined by self-reported country of birth or birth in one of the Moluccan resettlement camps.

Regarding socio-demographic variables, socio-economic status (SES) was determined using the Netherlands Institute for Social Research (SCP) status scores that are based upon the social status of a particular postcode area [15]. The number of years of education was measured by the sum of years of education in the country of origin and in the Netherlands. Age, gender, and marital status were self-reported. The number of years of residence in the Netherlands was determined from the year of immigration to the Netherlands. Language proficiency 
was measured by self-reported difficulty in speaking Dutch. Acculturation was measured using the adapted Psychological Acculturation Scale (PAS) [16], originally developed by Tropp et al. [17]. The PAS consists of two scales evaluating emotional attachment to Dutch culture (D-PAS) and culture of origin (in this study defined as C-PAS). Multimorbidity was defined as the presence of two or more chronic conditions from a selection of 17 self-reported chronic conditions. Loneliness was measured by the De Jong Gierveld loneliness scale [18].

\section{Data analysis}

We compared the three ethnic groups with respect to socio-demographic information, health characteristics, and HRQOL. Chi square tests were performed to evaluate differences in nominal and ordinal variables. Analysis of variance (ANOVA) was used for continuous variables with the Welch modification when the assumption of homogeneity of variance was not met. A significant ANOVA was followed by post hoc multiple comparison testing using Bonferroni's test. The Games-Howell test was used when the assumption of homogeneity of variance was violated.

Psychometric properties of the translated instruments were absent for the immigrant groups in our study. We therefore assessed Cronbach's alpha in the total population and for the three immigrant groups separately.

Hierarchical multiple regressions were executed for PCS and MCS to examine whether ethnicity contributed uniquely to the variance in HRQOL when multimorbidity, loneliness, socio-demographics, and acculturation were taken into account. Correlations lower than 0.80 , suggesting that multicollinearity was at an acceptable level, permitted the entry of the variables [19]. Independent variables were entered into five blocks in the hierarchical multiple regression. In the first block, PCS was regressed on ethnicity only. In the second block, PCS was regressed on multimorbidity, in addition to ethnicity. In third block, loneliness was added. In the fourth block, acculturation, years of residence in the Netherlands, and language proficiency were entered. In the final block, age, gender, SES, years of education, and marital status were added. An identical regression analysis was performed with MCS as the dependent variable.

Statistical significance level was set at 0.05. All analyses were performed in SPSS 20.0 for Windows.

\section{Results \\ Sample}

A total of 478 elderly immigrants were approached for participation in the study, and 201 completed the questionnaire. The overall response rate was $42.1 \%$ (38.1\% among Moroccans, $79.3 \%$ among Turks, and 25.4\% among Moluccans).
As shown in Table 1 , the mean $( \pm \mathrm{SD})$ age of the Turks was $63.3 \pm 5.0$; of the Moroccans was $67.1 \pm 7.3$; and of the Moluccans was $69.7 \pm 8.6$. The average number of years of residence in the Netherlands was $59.4 \pm 4.8$ for the Moluccans, $34.7 \pm 10.6$ for the Moroccans, and $34.2 \pm$ 8.1 for the Turks. A high proportion of Moroccans (100\%) and Turks (89.9\%) showed an SES below the Dutch average. Additionally, $76.8 \%$ of the Turks, $57.1 \%$ of the Moroccans, and $44.1 \%$ of the Moluccans reported multimorbidity. Moroccans scored highest on loneliness (7.3 \pm 4.2$)$, followed by Turks ( $5.4 \pm 3.8)$, and Moluccans $(1.6 \pm 2.0)$.

\section{Psychometric properties}

The overall Cronbach's alpha $\alpha$ was 0.89 for PCS $(0.80$ for Moroccans, 0.82 for Turks, and 0.75 for Moluccans) and 0.82 for MCS ( 0.90 for Moroccans, 0.88 for Turks, and 0.77 for Moluccans). Overall Cronbach's alpha $\alpha$ was 0.79 for the D-PAS ( 0.86 for Moroccans, 0.75 for Turks, and 0.85 for Moluccans) and 0.85 for the C-PAS (0.91 for Moroccans, 0.70 for Turks, and 0.70 for Moluccans). Cronbach's alpha $\alpha$ was 0.92 for the loneliness scale (0.93 for Moroccans, 0.91 for Turks, and 0.69 for Moluccans).

\section{Sample characteristics}

Table 1 shows that differences among the ethnic groups were found in the categories of years of education $(p<$ $0.05)$, language proficiency $(p<0.05)$, loneliness $(p<0.05)$, and HRQOL $(p<0.05)$. Regarding HRQOL, Moroccans had the lowest scores on PCS $(34.3 \pm 31.4)$ and MCS (42.1 \pm 27.0 ), followed by Turks ( $45.7 \pm 27.0$ for PCS and $54.7 \pm 22.2$ for MCS), and Moluccans $(71.7 \pm 21.2$ for PCS and $74.4 \pm 22.1$ for MCS).

\section{Determinants of HRQOL}

Multiple regression analysis (Table 2, Model 5) showed that ethnicity was not independently associated with PCS and MCS, in contrast to loneliness (PCS $\beta-0.461$, $p<0.001$ and MCS $\beta-0.435, p<0.001)$ and multimorbidity (PCS $\beta-0.380, p<0.001$ and MCS $\beta-0.398, p<$ $0.001)$. Gender was independently associated with PCS ( $\beta$ 0.148, $p=0.026)$ and attachment to Dutch culture with MCS ( $\beta$ 0.144, $p=0.029)$.

\section{Discussion}

The present study revealed that elderly immigrant populations in the Netherlands experience different levels of HRQOL, but these differences are not related to ethnic background. Multimorbidity and loneliness counted for most of the differences in HRQOL. In addition, gender and attachment to Dutch culture moderately contributed to the variance in HRQOL. These outcomes suggest that elderly from different ethnic backgrounds have a more 
Table 1 Socio-demographic and health characteristics of the study sample

\begin{tabular}{|c|c|c|c|c|}
\hline & Moroccan & Turkish & Moluccan & $p$-value \\
\hline & $\mathrm{N}=98$ & $N=69$ & $\mathrm{~N}=34$ & \\
\hline Age (mean, SD) & $67.1(7.3)^{1}$ & $63.3(5.0)^{2}$ & $69.7(8.6)^{3}$ & $<0.001$ \\
\hline Age (\%) & & & & 0.001 \\
\hline $55-59$ years & 18.4 & 27.5 & 14.7 & \\
\hline $60-69$ years & 41.8 & 60.9 & 38.2 & \\
\hline$\geq 70$ years & 39.8 & 11.6 & 47.1 & \\
\hline Gender (\%) & & & & 0.724 \\
\hline Male & 56.1 & 50.7 & 50.0 & \\
\hline Years of residence in the Netherlands ${ }^{a}$ (mean, SD) & $34.7(10.6)^{4}$ & $34.2(8.1)^{3}$ & $59.4(4.8)^{2}$ & $<0.001$ \\
\hline Years of education (mean, SD) & $1.1(3.0)^{2}$ & $3.7(3.2)^{2}$ & $10.4(4.3)^{2}$ & $<0.001$ \\
\hline SES below Dutch average ${ }^{\mathrm{b}}(\%)$ & 100 & 89.9 & 55.9 & $<0.001$ \\
\hline Marital status (\%) & & & & 0.529 \\
\hline Married & 82.3 & 88.4 & 82.4 & \\
\hline Divorced/widowed & 17.7 & 11.6 & 17.6 & \\
\hline \multicolumn{5}{|l|}{ Acculturation (PAS) } \\
\hline D-PAS $($ mean, SD) & $19.3(4.6)$ & $18.8(5.4)^{3}$ & $21.9(6.2)^{3}$ & 0.048 \\
\hline C-PAS ${ }^{d}$ (mean, SD) & $23.1(5.1)^{2}$ & $26.9(3.8)^{1}$ & $26.2(3.8)^{4}$ & $<0.001$ \\
\hline Language proficiency (mean, SD) & $2.7(0.8)^{2}$ & $2.3(0.5)^{2}$ & $3.6(0.6)^{2}$ & $<0.001$ \\
\hline Multimorbidity (\%) & & & & 0.004 \\
\hline 0 chronic conditions & 20.4 & 10.1 & 38.2 & \\
\hline 1 chronic condition & 22.4 & 13.0 & 17.6 & \\
\hline$\geq 2$ chronic conditions & 57.1 & 76.8 & 44.1 & \\
\hline Loneliness (De Jong Gierveld & $7.3(4.2)^{2}$ & $5.4(3.8)^{2}$ & $1.6(2.0)^{2}$ & $<0.001$ \\
\hline \multicolumn{5}{|l|}{ loneliness scale) $)^{f}$ (mean, SD) } \\
\hline \multicolumn{5}{|l|}{ HRQOL (SF-12) } \\
\hline $\mathrm{PCS}^{9}($ mean, SD) & $34.3(31.4)^{2}$ & $45.7(27.0)^{2}$ & $71.7(21.2)^{2}$ & $<0.001$ \\
\hline $\mathrm{MCS}^{\mathrm{h}}($ mean, SD) & $42.1(27.0)^{2}$ & $54.7(22.2)^{2}$ & $74.4(22.1)^{2}$ & $<0.001$ \\
\hline
\end{tabular}

${ }^{a}$ Moluccans born in the Netherlands were not asked.

${ }^{b}$ Status scores were classified into SES above the Dutch average and below the Dutch average. The average status score in the Netherlands was used as cut-off point.

${ }^{\mathrm{C}}$ Ranged from 6 to 30. A higher score means a greater sense of emotional attachment and belonging within the Dutch culture.

${ }^{d}$ Ranged from 6 to 30. A higher score means greater sense of emotional attachment and belonging within the culture of origin

${ }^{\mathrm{e}}$ Ranged from 1 to 4 . A higher score means a better command of the Dutch language.

${ }^{f}$ Ranged from 0 to 11. A higher score indicates a greater experience of feelings of loneliness.

${ }^{9}$ Ranged from 0 to 100 . A higher score indicates better physical health.

${ }^{\mathrm{h}}$ Ranged from 0 to 100 . A higher score indicates better mental health.

${ }^{1}$ Means of Moroccans and Turks differ significantly $(p<0.05)$.

${ }^{2}$ Means of Moroccans, Turks, and Moluccans differ significantly $(p<0.05)$

${ }^{3}$ Means of Moluccans and Turks differ significantly $(p<0.05)$.

${ }^{4}$ Means of Moluccans and Moroccans differ significantly $(p<0.05)$.

or less comparable level of HRQOL as long as their health status, social resources, and level of acculturation are comparable.

Within the different ethnic groups, Moluccans had, by far, the best HRQOL, whereas Moroccans had the poorest. The Turks scored in between these two groups. This trend was evident in both the physical and mental aspects of quality of life. In addition to our findings that multimorbidity and loneliness are important predictive factors, we performed additional analyses for the mean scores of
HRQOL adjusted for these two factors. After adjustment, average physical and mental quality of life improved slightly for Moroccans and Turks, but not for Moluccans (adjusted mean PCS scores: Moroccans 38.8, Turks 47.7, Moluccans 53.0; adjusted mean MCS scores: Moroccans 45.7, Turks 55.2, Moluccans 59.5). The Moroccans, in contrast to the Turks, even after adjustment still showed significantly worse HRQOL than the Moluccans.

Our results raise the question: Does the mean score of older immigrants differ from that of the native Dutch 
Table 2 PCS and MCS assessed by linear regression analysis ( $\beta^{\prime} \mathbf{s}$ )

\begin{tabular}{|c|c|c|c|c|c|c|c|c|c|c|}
\hline \multirow[t]{4}{*}{ Physical Component Summary (PCS) } & \multirow{2}{*}{\multicolumn{2}{|c|}{$\begin{array}{l}\text { Model } 1 \\
\mathrm{R}^{2} \text { adj. } 0.114\end{array}$}} & \multirow{2}{*}{\multicolumn{2}{|c|}{$\frac{\text { Model } 2}{\mathrm{R}^{2} \text { adj. } 0.335}$}} & \multirow{2}{*}{\multicolumn{2}{|c|}{$\frac{\text { Model } 3}{\mathrm{R}^{2} \text { adj. } 0.506}$}} & \multirow{2}{*}{\multicolumn{2}{|c|}{$\frac{\text { Model } 4}{\mathrm{R}^{2} \text { adj. } 0.516}$}} & \multirow{2}{*}{\multicolumn{2}{|c|}{$\begin{array}{l}\text { Model } 5 \\
\mathrm{R}^{2} \text { adj. } 0.538\end{array}$}} \\
\hline & & & & & & & & & & \\
\hline & \multicolumn{2}{|c|}{$\begin{array}{l}F 11.624 \\
(p<0.001)\end{array}$} & \multicolumn{2}{|c|}{$\begin{array}{l}F 28.711 \\
(p<0.001)\end{array}$} & \multicolumn{2}{|c|}{$\begin{array}{l}F 43.286 \\
(p<0.001)\end{array}$} & \multicolumn{2}{|c|}{$\begin{array}{l}F 23.027 \\
(p<0.001)\end{array}$} & \multicolumn{2}{|c|}{$\begin{array}{l}F 15.775 \\
(p<0.001)\end{array}$} \\
\hline & $\beta$ & $p$-value & $\beta$ & $p$-value & $\beta$ & $p$-value & B & $p$-value & $\beta$ & $p$-value \\
\hline \multicolumn{11}{|l|}{ Ethnic group (vs. Moluccans) } \\
\hline Turkish & -0.396 & 0.001 & -0.321 & 0.001 & -0.140 & 0.115 & 0.040 & 0.731 & 0.033 & 0.803 \\
\hline Moroccan & -0.546 & $<0.001$ & -0.564 & $<0.001$ & -0.251 & 0.009 & -0.093 & 0.430 & -0.050 & 0.735 \\
\hline Multimorbidity (vs. no multimorbidity) & & & -0.480 & $<0.001$ & -0.395 & $<0.001$ & -0.395 & $<0.001$ & -0.380 & $<0.001$ \\
\hline Loneliness & & & & & -0.469 & $<0.001$ & -0.454 & $<0.001$ & -0.461 & $<0.001$ \\
\hline Years of residence in the Netherlands & & & & & & & 0.132 & 0.081 & 0.088 & 0.359 \\
\hline Dutch-PAS & & & & & & & 0.068 & 0.291 & 0.033 & 0.616 \\
\hline Country of origin-PAS & & & & & & & 0.005 & 0.935 & 0.010 & 0.871 \\
\hline Language proficiency & & & & & & & 0.047 & 0.483 & 0.022 & 0.740 \\
\hline Age & & & & & & & & & -0.042 & 0.553 \\
\hline Male (vs. female) & & & & & & & & & 0.148 & 0.026 \\
\hline SES below Dutch average (vs. above Dutch average) & & & & & & & & & -0.022 & 0.712 \\
\hline Education years & & & & & & & & & 0.119 & 0.130 \\
\hline Married (vs. divorced/widowed) & & & & & & & & & -0.102 & 0.081 \\
\hline \multirow[t]{4}{*}{ Mental Component Summary (MCS) } & \multicolumn{2}{|c|}{ Model 1} & \multicolumn{2}{|c|}{ Model 2} & \multicolumn{2}{|c|}{ Model 3} & \multicolumn{2}{|c|}{ Model 4} & \multicolumn{2}{|c|}{ Model 5} \\
\hline & \multicolumn{2}{|c|}{$\mathrm{R}^{2}$ adj. 0.117} & \multicolumn{2}{|c|}{$\mathrm{R}^{2}$ adj. 0.319} & \multicolumn{2}{|c|}{$\mathrm{R}^{2}$ adj. 0.480} & \multicolumn{2}{|c|}{$\mathrm{R}^{2}$ adj. 0.527} & \multicolumn{2}{|c|}{$\mathrm{R}^{2}$ adj. 0.529} \\
\hline & \multicolumn{2}{|c|}{$\begin{array}{l}F 11.891 \\
(p<0.001)\end{array}$} & \multicolumn{2}{|c|}{$\begin{array}{l}F 26.767 \\
(p<0.001)\end{array}$} & \multicolumn{2}{|c|}{$\begin{array}{l}F 39.085 \\
(p<0.001)\end{array}$} & $\begin{array}{l}F 23.95 \\
(p<0.0\end{array}$ & & $\begin{array}{l}F 15.23 \\
(p<0.0 \\
\end{array}$ & \\
\hline & $\beta$ & $p$-value & $\beta$ & $p$-value & $\beta$ & $p$-value & B & $p$-value & $\beta$ & $p$-value \\
\hline Ethnic group (vs. Moluccans) & & & & & & & & & & \\
\hline Turkish & -0.363 & 0.002 & -0.319 & 0.002 & -0.149 & 0.112 & 0.092 & 0.435 & 0.005 & 0.970 \\
\hline Moroccan & -0.560 & $<0.001$ & -0.606 & $<0.001$ & -0.304 & 0.003 & -0.112 & 0.343 & -0.240 & 0.114 \\
\hline Multimorbidity (vs. no multimorbidity) & & & -0.460 & $<0.001$ & -0.369 & $<0.001$ & -0.376 & $<0.001$ & -0.398 & $<0.001$ \\
\hline Loneliness & & & & & -0.453 & $<0.001$ & -0.425 & $<0.001$ & -0.435 & $<0.001$ \\
\hline Years of residence in the Netherlands & & & & & & & 0.118 & 0.107 & 0.018 & 0.853 \\
\hline Dutch-PAS & & & & & & & 0.156 & 0.014 & 0.144 & 0.029 \\
\hline Country of origin-PAS & & & & & & & 0.008 & 0.902 & 0.009 & 0.881 \\
\hline Language proficiency & & & & & & & 0.104 & 0.113 & 0.121 & 0.070 \\
\hline Age & & & & & & & & & 0.056 & 0.439 \\
\hline Male (vs. female) & & & & & & & & & 0.124 & 0.068 \\
\hline SES below Dutch average (vs. above Dutch average) & & & & & & & & & 0.071 & 0.236 \\
\hline Education years & & & & & & & & & 0.003 & 0.971 \\
\hline Married (vs. divorced/widowed) & & & & & & & & & -0.035 & 0.556 \\
\hline
\end{tabular}

older population? Comparison with available data on the average PCS and MCS scores for elderly from the native Dutch population in 2012 [20] showed that Moroccans and to a lesser extent Turks had a worse score on PCS than the Dutch (55-65 years 48.6; 65-75 years 47.4; $\geq 75$ years 42.4). The Moluccans had a better physical and mental quality of life. Moroccans had a worse score on MCS than the Dutch (55-65 years 53.5 ; 65-75 years
$55.0 ; \geq 75$ years 54.0), Turks had a quite comparable score, and Moluccans had a better one. In general, with the exception of the Moluccans, we can conclude that HRQOL, particularly physical quality of health was poorer than the native Dutch elderly population.

Our results did not completely resemble earlier research. In line with Schellingerhout [1], Moluccans reported the best mental quality of life. Schellingerhout [1] 
and Denktaş [2] found no differences between Turks and Moroccans. However, in our study, Turks and Moroccans experienced different levels of HRQOL with Moroccans being the most disadvantaged. Differences in area of residence could explain discrepancies in HRQOL. The Turks in our study lived in a semi-urban setting. However, the Moroccans in our study and the Moroccans and Turks in the studies by Schellingerhout and Denktaş lived in deprived neighbourhoods in a large city. The literature suggests that poorer health status and harmful health behaviour occur more often in deprived neighbourhoods [21].

That multimorbidity as an important predictive factor for low HRQOL is consistent with research showing that chronic conditions and multiple morbidities are associated to poorer HRQOL $[9,10]$. Our study confirms this for elderly immigrants. Our results also confirm that loneliness is highly associated with low HRQOL in the elderly [8,22], but the causal relation can be interpreted in two different ways. On the one hand, a low HRQOL may lead to loss of social interactions and, ultimately, to feelings of loneliness [11]. On the other hand, loneliness may lead to experiencing poor HRQOL.

We found gender to be associated with physical quality of life, which is consistent with previous studies showing a better HRQOL for men, regardless of whether they were natives or immigrants [10-12]. As we corrected for age and multimorbidity these factors are not a plausible explanation. The association is more likely to be explained by the fact that women have a more acute perception of their health problems and consequently they are more likely to report them [12].

Our finding that attachment to Dutch culture is associated with a higher mental quality of life is in contrast to earlier research. Schellingerhout found no influence of acculturation [1]. This discrepancy might be due to differences in the acculturation measures used in the different studies. Schellingerhout used variables such as Dutch language use, informal contacts with native Dutch, and attitudes on care and family values as indicators of acculturation, whereas we focussed on individuals' psychological identification with Dutch culture and the subjects' cultures of origin. Nevertheless, it is quite understandable that elderly who are more emotionally attached to and integrated with Dutch people and culture perceive a better quality of life.

There are some limitations to our study that need to be considered when interpreting our results. First, although the SF-36, the parent questionnaire for the SF12 , and the SF-12 itself are widely used valid instruments that have been previously used to measure HRQOL including immigrants $[1,2,6,12,23]$, measurement bias might have been occurred by the blending of health and physical and mental function concepts in the SF-36
$[24,25]$. As our study focused on the physical and mental health summary scores, it could still be possible that ethnicity affected HRQOL independently of physical and mental functioning. To exclude this possibility, additional analyses were performed on the SF-12 general health rating item ("In general how would you rate your health?"), which is in contrary to the other SF-12 items not specifically related to physical and mental function. It is one of the most frequently asked questions to assess health status. In line with our findings on the PCS and MCS, ethnicity was not associated with this single SF-12 item. This suggests that even independently of physical and mental functioning, ethnicity seems not to affect HRQOL.

A second limitation was the non-random sampling of the study participants. Given the often poor response in migrant groups and the risk that the role of CHWs in recruiting participants in a strictly randomised design conflicts with their role as community health advocates [26], we believed that random sampling was not appropriate for our study. This has most likely not biased the selection of the study population because approximately half of the total number of elderly from each ethnicity, approximately $42.9 \%$ Turks, $46.6 \%$ Moroccans, and 67.3\% Moluccans, who were approached participated in the study.

A third limitation was the unbalanced response rate. The overall response rate of $42.1 \%$ was acceptable and comparable with previous research among elderly immigrants (48\%) [1]. However, as in the study by Schellingerhout (65.3\% Moroccans, 43.6\% Turks, Moluccans not reported) [1], the response rate in our study varied strongly across the three ethnic groups (38.1\% Moroccans, $79.3 \%$ Turks, and 25.4\% Moluccans). A non-response analysis showed no selection according to age and gender for Moroccans and Turks. Selection for Moluccans was found regarding age (a lower average age among the nonparticipants: 63.8 versus 69.7) but not gender. Because our findings are consistent with previous research [1], this, as well as the small number of Moluccans, has not likely influenced the results for Moluccan participants. Another possible risk for bias was that we, due to the limited number of first generation Moluccan immigrants, also included descendants of Moluccan immigrants born and raised in resettlement camps in the Netherlands. We compared Moluccan immigrants with descendants born in the resettlement camps and found that no differences between the two groups, except of course for age, existed in socio-demographic features, health characteristics and HRQOL.

Fourth, not all measuring instruments were available in the appropriate language for the immigrant groups studied, and they were therefore translated. We presented Cronbach's alpha in the total population and for 
the three immigrant groups separately for PCS, MCS, D-PAS, C-PAS, and the De Jong Gierveld loneliness scale. These scales showed a good to acceptable reliability in all three groups, with alphas varying from 0.69 to 0.93 . We can conclude that these instruments are appropriate for the immigrant groups.

Finally, the self-reported data may have resulted in either report or recall bias. Previous research on HRQOL [6] determined that cross-cultural differences in health perceptions might influence self-reported HRQOL. One might hypothesise that the conceptualisation of health and quality of life depends on cross-cultural differences. Because we found that the PCS and MCS were highly reliable $(0.75-0.90)$, we conclude that the underlying structure of the concept of quality of life is the same for all three immigrant groups.

\section{Conclusion}

Multimorbidity and loneliness, rather than ethnicity, determine the level of HRQOL reported by elderly immigrant populations. Our results suggest that health and prevention programmes meant to improve HRQOL within the ethnic groups do not need to be specific for a particular elderly immigrant population but, rather, should address loneliness and multimorbidity. A potential successful approach to improve HRQOL, for both immigrant and native elderly, could be to screen for developing loneliness and multimorbidity. This could be particularly supportive for Moroccan and Turkish elderly who are most at risk. In addition, interventions addressing loneliness and chronic diseases should be provided as a regular part of usual care. But additional interventions when meant to remove HRQOL differences between the ethnic groups are needed in case of Moroccan and Moluccan elderly. Finally, health literacy deserves attention as immigrants often lack the requisite health literacy skills to maintain health [27]. Therefore culturally sensitive communication programmes are needed for providing immigrant elderly with the health information they need to accessing and making sense of relevant health information.

\section{Competing interests}

The authors declare that they have no competing interests.

\section{Authors' contributions}

NW, WR and BS designed the study and wrote the grant application. IV drafted the manuscript. WR, NW and BS commented on draft versions of the paper. Al authors read and approved the final manuscript.

\section{Acknowledgements}

This study is funded by a grant from ZonMw, the Netherlands Organisation for Health Research and Development (ZonMw, reference 314040201).

Received: 22 January 2014 Accepted: 26 August 2014

Published online: 01 October 2014

\section{References}

1. Schellingerhout R: Gezondheid en Welzijn van allochtone ouderen [Social Position, Health and Well-being of Elderly Immigrants]. Den Haag: Sociaal en Cultureel Planbureau; 2004

2. Denktaş S: Health and Health Care Use of Elderly Immigrants in the Netherlands. A comparative study. PhD thesis. In Erasmus University Rotterdam; 2011.

3. van der Wurff FB, Beekman ATF, Dijkshoorn H, Spijker JA, Smits CHM, Stek $M L$, Verhoeff A: Prevalence and risk-factors for depression in elderly Turkish and Moroccan migrants in the Netherlands. J Affect Disord 2004, 83:33-41.

4. Silveira E, Skoog I, Sundh V, Allebeck P, Steen B: Health and well-being among 70-year-old migrants living in Sweden-results from the $\mathrm{H} 70$ gerontological and geriatric population studies in Göteborg. Soc Psychiatry Psychiatr Epidemiol 2002, 37:13-22.

5. Pudaric S, Sundquist J, Johansson SE: Country of birth, instrumental activities of daily living, self-rated health and mortality: a Swedish population-based survey of people aged 55-74. Soc Sci Med 2003, 56:2493-2503.

6. Koochek A, Montazeri A, Johansson SE, Sundquist J: Health-related quality of life and migration: A cross-sectional study on elderly Iranians in Sweden. Health Qual Life Outcomes 2007, 5:60.

7. Bowling A, Banister D, Sutton S, Evans O, Windsor J: A multidimensional model of the quality of life in older age. Aging Mental Health 2002, 6:355-371.

8. Ekwall AK, Sivberg B, Hallberg IR: Loneliness as a predictor of quality of life among older caregivers. J Adv Nurs 2005, 49:23-32.

9. Mier N, Ory MG, Zhan D, Conkling M, Sharkey JR, Burdine JN: Health-related quality of life among Mexican Americans living in colonias at the TexasMexico border. Soc Sci Med 2008, 66:1760-1771.

10. Orfila F, Ferrer M, Lamarca R, Tebe C, Domingo-Salvany A, Alonso J: Gender differences in health-related quality of life among the elderly: the role of objective functional capacity and chronic conditions. Soc Sci Med 2006, 63:2367-2380

11. Gallicchio L, Hoffman SC, Helzlsouer KJ: The relationship between gender, social support, and health-related quality of life in a community-based study in Washington County, Maryland. Qual Life Res 2007, 16:777-786.

12. Daher AM, Ibrahim HS, Daher TM, Anbori AK: Health related quality of life among Iraqi immigrants settled in Malaysia. BMC Public Health 2011, 11:407.

13. Verhagen I, Ros WJG, Steunenberg B, de Wit NJ: Culturally sensitive care for elderly immigrants through ethnic community health workers: design and development of a community based intervention programme in the Netherlands. BMC Public Health 2013, 13:227.

14. Ware JE, Kosinksi M, Keller SD: SF-12: How to Score the SF-12 Physical and Mental Health Summary Scales. Boston: The Health Institute, New England Medical Center; 1995.

15. Knol F: Stadsontwikkeling van wijken in Nederland 1998-2010 [Neighbourhood Status Development in the Netherlands 1998-2010]. Den Haag: Sociaal en Cultureel Planbureau; 2012.

16. Stevens GWJM, Pels TVM, Vollebergh WAM, Crijnen AAM: Patterns of Psychological Acculturation in Adult and Adolescent Moroccan Immigrants Living in the Netherlands. J Cross-Cult Psychol 2004, 35:689-704

17. Tropp LR, Erkut S, Coll CG, Alarcón O, Vázquez García HA: Psychological acculturation: Development of a new measure for Puerto Ricans on the U.S. mainland. Educ Psychol Meas 1999, 59:351-367.

18. de Jong GJ, van Tilburg TG: Manual of the Loneliness Scale. Amsterdam: Vrije Universiteit Amsterdam, Department of Social Research Methodology; 1999.

19. Field A: Discovering Statistics Using SPSS. London: SAGE Publications Ltd; 2009.

20. CBS [The Central Bureau of Statistics]: Gezondheidsmonitor 2012 [Health Monitor 2012]. Voorburg/Heerlen: CBS; 2012.

21. Reijneveld SA: Neighbourhood socioeconomic context and self reported health and smoking: a secondary analysis of data on seven cities. J Epidemiol Community Health 2002, 56:935-942.

22. Luanaigh CO, Lawlor BA: Loneliness and the health of older people. Int $J$ Geriatric Psychiatry 2008, 23:1213-1221.

23. Domnich A, Amicizia D, Panatto D, Signori A, Perelli V, Adanoli S, Riboli EB, Gasparini R: Use of different subjective health indicators to assess health inequalities in an urban immigrant population in north-western Italy: a cross-sectional study. BMC Public Health 2013, 13:1006 
24. Hays RD, Hahn H, Marshall G: Use of the SF-36 and other health-related quality of life measures to assess persons with disabilities. Arch Psych Med Rehabil 2002, 83(Suppl 2):S4-S9.

25. Horner-Johnson W, Krahn GL, Suzuki R, Peterson JJ, Roid G, Hall T, the RRTC Expert Panel on Health Measurement: Differential performance of SF-36 items in healthy adults with and without functional limitations. Arch Psych Med Rehabil 2010, 91:570-575.

26. Terpstra J, Coleman KJ, Simon G, Nebeker C: The role of community health workers (CHWs) in health promotion research: ethical challenges and practical solutions. Health Promot Pract 2011, 12:86-93.

27. Kreps GL, Sparks L: Meeting the health literacy needs of immigrant populations. Patient Educ Couns 2008, 71:328-332.

doi:10.1186/s12955-014-0138-8

Cite this article as: Verhagen et al: Ethnicity does not account for differences in the health-related quality of life of Turkish, Moroccan, and Moluccan elderly in the Netherlands. Health and Quality of Life Outcomes 2014 12:138

\section{Submit your next manuscript to BioMed Central and take full advantage of:}

- Convenient online submission

- Thorough peer review

- No space constraints or color figure charges

- Immediate publication on acceptance

- Inclusion in PubMed, CAS, Scopus and Google Scholar

- Research which is freely available for redistribution 